\title{
Peculiarities of Propagation of Electromagnetic Excitations through Nonideal 1d Photonic Crystal
}

Vladimir V. Rumyantsev*

A.A. Galkin Donetsk Institute for Physics and Engineering, National Academy of Sciences, Ukraine

\begin{abstract}
Nonideal 1D photonic crystal is modeled as a macroscopically homogeneous layered system (which is onedimensional Si-liquid crystal superlattice with two elements-layers in the cell) with randomly included admixture layers. The virtual crystal approach which is the method to describe quasi-particle excitations in disorder media is used. Peculiarities of the dependence of photonic band gap width on admixture layers concentration have been studied. The results are the evidence of substantial polariton spectrum reconstruction caused by presence of defect layers.
\end{abstract}

Keywords: 1D photonic crystal; Admixture elements; Virtual crystal approximation; Band gap width

\section{Introduction}

At present, propagation of electromagnetic waves in thin films and layered crystal media [1-4], in particular, in photonic magnetic crystals [5-7] and composite crystals based on silicon and liquid crystal [8-12], are being investigated extensively. Interest in investigation of these objects is motivated, on the one hand, by the demand for different layered structures with given properties in solid-state electronics and, on the other hand, by achievements in technologies providing the possibility of growing such films and periodic structures with controlled characteristics by the molecular-beam epitaxial method. A large number of works [13-15] have been devoted to theoretical and experimental investigation of exciton-type excitations in dielectric ideal superlattices. The general theory of optical waves in anisotropic crystals, including those composed of macroscopic layers, is considered in [16]. In [11], the forbidden photonic bands of a crystal made of alternating silicon and liquid crystal layers are calculated. 1D photonic band gap structures attract lot of attention of researches due to their omnifarious using for optical filtering [17], sensing [18] and so on. The logic of further development of the theory of layered structures requires consideration of more complex systems-superlattices with impurity layers, as well as with layers of variable composition and thickness. In [6], the dispersion of polaritons in a superlattice with a single impurity layer has been studied. At the same time, of considerable interest are investigations of nonideal superlattices with an arbitrary number of impurity layers and of the dependence of the polariton spectrum on the concentration of corresponding defects, which make it possible to expand the capabilities of modeling the properties of such systems and to create layered materials with given characteristics.

The method of calculation of polariton excitations has much in common with methods of finding other quasiparticle excitations (electron, phonon, etc.) in solids. In the present chapter authors offer for description of photon modes in macroscopically heterogeneous ambience approach, based on configuration averaging (it specifically is new in this case), which was used before [19] for the microscopic calculation of disordered systems quasiparticles spectra. A relatively simple approximation in framework of this approach for calculation polariton spectra and corresponding optical characteristics of disordered ambiences is the Virtual-Crystal Approximation (VCA) [19-21]. VCA (first it was used by Nordheim and Parmenter [19]) consist of replacing of correct one-electron potential (appropriate to a given configuration of atoms of the alloy) by its average which is taken over all possible random configurations. The approximation is a widely used for study of disordered structures. For example, based on the pseudopotential scheme under the VCA in which the effect of compositional disorder is involved, the dependence of optoelectronic properties of $\mathrm{GaAs}_{x} \mathrm{Sb}_{1-x}$ on alloy composition $x$ have been studied in [22]. Within this approximation the configurationally dependent parameters of the Hamiltonian are replaced with their configurationally averaged values. Description of transformation of a polariton spectrum in a sufficiently simple superlattice, using VCA, is the first step towards the study of imperfect systems. However investigation of properties of polariton spectra and the related physical quantities (density of elementary excitation states, characteristics of the normal electromagnetic waves etc.) in less simple systems requires application of more complex methods. Such are the method of the coherent (oneor many-site) potential [21], the averaged T-matrix method [23] and their numerous modifications used for various particular problems.

1D photonic crystal may be modeled as a set of macroscopically homogeneous layers with randomly included extrinsic (with respect to the ideal superlattice) layers. Corresponding configuration-dependent material tensors in our model of an imperfect superlattice are represented in terms of random quantities. After configuration-averaging the translational symmetry of a considered system is "restored" that allows us obtain the system of equations which define normal modes of electromagnetic waves, propagating in one-dimensional (1D) "periodic" medium. Within the VCA the peculiarities of the dependence of the band gap width and refractive index upon concentration of admixture layers for the non-ideal photonic crystalline system (that is layered structure or striped thin film) is studied by authors [24]. The photon modes spectrum of a non-ideal superlattice with an arbitrary number of layers (strips) per elementary cell, obtained within the VCA, is concretized for the Si - liquid crystal system. Dependence of the band gap width upon concentration of admixture elements and refractive index peculiarities was analyzed.

*Corresponding author: Vladimir V. Rumyantsev, A.A. Galkin Donetsk Institute for Physics and Engineering, National Academy of Sciences, Ukraine, E-mail: rumyants@teor.fti.ac.donetsk.ua

Received May 27, 2013; Accepted July 23, 2013; Published July 25, 2013

Citation: Rumyantsev VV, Schwartzman A (2013) Peculiarities of Propagation of Electromagnetic Excitations through Nonideal 1d Photonic Crystal. J Electr Electron Syst 2: 109. doi:10.4172/2332-0796.1000109

Copyright: () 2013 Rumyantsev VV, et al. This is an open-access article distributed under the terms of the Creative Commons Attribution License, which permits unrestricted use, distribution, and reproduction in any medium, provided the original author and source are credited. 


\section{Propagation of Electromagnetic Waves in Nonhomoge- neous Structures}

Dielectric $\hat{\varepsilon}(\vec{r})$ and magnetic $\hat{\mu}(\vec{r})$ permeability, which determine optical characteristics of a periodic medium, must satisfy the periodic boundary conditions:

$$
\hat{\varepsilon}(x, y, z)=\hat{\varepsilon}(x, y, z+d), \quad \hat{\mu}(x, y, z)=\hat{\mu}(x, y, z+d)
$$

where $d=\sum^{\sigma} a_{j}$ is the period of the superlattice, $\sigma$ is the number of layers per elementary cell, $a_{j}$ are the thicknesses of the layers which form a one-dimensional chain of elements oriented along the $z$-axis. The material tensors $\hat{\varepsilon}$ and $\hat{\mu}$ of a crystalline superlattice with an arbitrary number of layers $\sigma$ have the following form in the coordinate representation:

$$
\left(\begin{array}{l}
\hat{\varepsilon}(z) \\
\hat{\mu}(z)
\end{array}\right)=\sum_{n, \alpha}\left(\begin{array}{c}
\hat{\varepsilon}_{n \alpha} \\
\hat{\mu}_{n \alpha}
\end{array}\right)\left\{\theta\left[z-(n-1) d-\left(\sum_{j=1}^{\alpha} a_{n j}-a_{n \alpha}\right)\right]-\theta\left[z-(n-1) d-\sum_{j=1}^{\alpha} a_{n j}\right]\right\}
$$

In Eq. (2) $\theta(z)$ is the Heaviside function, $n= \pm 1, \pm 2, \ldots$ is the number of a one-dimensional crystal cell, index $\alpha=1,2, \ldots, \sigma$ designates the elements of the cell. Below we consider an imperfect system, in which disordering is connected with variation of the composition (rather than of the thickness) of admixture layers, so that $a_{n \alpha} \equiv a_{\alpha}$. Within our model, the configurationally dependent tensors $\hat{\varepsilon}_{n \alpha}, \hat{\mu}_{n \alpha}$ are expressed through the random quantities $\eta_{n \alpha}^{v}\left(\eta_{n \alpha}^{v}=1\right.$ if the $v(\alpha)$-th sort of layer is in the $(n \alpha)$-th site of the crystalline chain, $\eta_{n \alpha}^{v}=0$ otherwise):

$$
\left(\begin{array}{c}
\hat{\varepsilon}_{n \alpha} \\
\hat{\mu}_{n \alpha}
\end{array}\right)=\sum_{v(\alpha)}\left(\begin{array}{c}
\hat{\varepsilon}_{\alpha}^{v(\alpha)} \\
\hat{\mu}_{\alpha}^{v(\alpha)}
\end{array}\right) \eta_{n \alpha}^{v(\alpha)}
$$

Calculation of a polariton spectrum for the imperfect superlattice is realized within the VCA (similarly to the solid quasi-particle approach) through the following replacement: $\hat{\varepsilon} \rightarrow\langle\hat{\varepsilon}\rangle, \hat{\mu} \rightarrow\langle\hat{\mu}\rangle$ (angular parentheses designate the procedure of configuration averaging). In addition, from Eq. (3) and [21] we have:

$$
\left(\begin{array}{c}
\left\langle\hat{\varepsilon}_{n \alpha}\right\rangle \\
\left\langle\hat{\mu}_{n \alpha}\right\rangle
\end{array}\right)=\sum_{\alpha, v(\alpha)}\left(\begin{array}{c}
\hat{\varepsilon}_{\alpha}^{v(\alpha)} \\
\hat{\mu}_{\alpha}^{v(\alpha)}
\end{array}\right) C_{\alpha}^{v(\alpha)}
$$

where $C_{\alpha}^{\nu(\alpha)}$ is the concentration of the $v(\alpha)$-th sort of admixture layer in the $\alpha$-th sublattice. There is a normalization condition $\sum C_{\alpha}^{v(\alpha)}=1$. It follows from Eq. (2) that the Fourier-amplitudes $\hat{\varepsilon}_{l}, \hat{\mu}_{l}$ and the averaged dielectric $\left\langle\hat{\varepsilon}_{n \alpha}\right\rangle$ and magnetic $\left\langle\hat{\mu}_{n \alpha}\right\rangle$ permeabilities of layers (4) are related as

$$
\left(\begin{array}{c}
\hat{\varepsilon}_{l} \\
\hat{\mu}_{l}
\end{array}\right)=-\frac{i}{2 \pi l} \sum_{\alpha}\left(\begin{array}{l}
\left\langle\hat{\varepsilon}_{n \alpha}\right\rangle \\
\left\langle\hat{\mu}_{n \alpha}\right\rangle
\end{array}\right)\left\{\exp \left(i \frac{2 \pi}{d} l \sum_{j=1}^{\alpha} a_{j}\right)-\exp \left[i \frac{2 \pi}{d} l\left(\sum_{j=1}^{\alpha} a_{j}-a_{\alpha}\right)\right]\right\}
$$

Since the configurational averaging "restores" the translational symmetry of a crystalline system, in the considered case of imperfect superlattice the "acquired" translational invariance of the onedimensional chain allows us to present Maxwell equations (for harmonic dependency of the electric and magnetic field strengths $\vec{E}(\vec{r}, \omega), \vec{H}(\vec{r}, \omega)$ on a time) in the form:

$$
\nabla \times \vec{E}(\vec{r}, \omega)=\frac{i \omega}{c}\langle\hat{\mu}(z)\rangle \cdot \vec{H}(\vec{r}, \omega), \quad \nabla \times \vec{H}(\vec{r}, \omega)=-\frac{i \omega}{c}\langle\hat{\varepsilon}(z)\rangle \cdot \vec{E}(\vec{r}, \omega)
$$

Hence, according to the Floquet theorem, Fourier-amplitudes $\vec{f}_{K, p}^{(E, H)}$ of the electric and magnetic field strengths satisfy the following relation:

$$
\left[\vec{\beta}+\left(K+p \frac{2 \pi}{d}\right) \vec{e}_{z}\right] \times\left(\begin{array}{l}
\vec{f}_{K, p}^{(H)} \\
\vec{f}_{K, p}^{(K)}
\end{array}\right)=\frac{\omega}{c}\left[\begin{array}{c}
-\sum_{l} \hat{\varepsilon}_{l} \cdot \vec{f}_{K, p-l}^{(E)} \\
\sum_{l} \hat{\mu}_{l} \cdot \vec{f}_{K, p-l}^{(H)}
\end{array}\right]
$$

Here $\vec{\beta}$ is an arbitrary planar (in the XOY plane) wave vector, $\vec{e}_{z}$ is a unit vector along the $z$-axis, $\vec{K}=(0,0, K)$ is the Bloch vector. The system
(7) defines normal modes of electromagnetic waves, propagating in the considered "periodic" medium. Below, for simplicity, we shall restrict our study to the case of light, propagating along the $z$-axis $(\vec{\beta}=0)$ in a nonmagnetic lattice, $\hat{\mu}=\hat{I}$ is a unit matrix; the liquid-crystal layers we shall treat as uniaxial, $\varepsilon_{\dot{j}}=\varepsilon_{x} \delta_{\dot{x}} \delta_{\dot{k}}+\varepsilon_{y} \delta_{\dot{y}} \delta_{\dot{j}}+\varepsilon_{z} \delta_{\dot{i}} \delta_{\dot{k}}$; obviously, that for $\vec{K} \mid z, z z$-components of the tensor $\hat{\varepsilon}$ do not appear in final formulas, and $\varepsilon_{x}=\varepsilon_{y} \equiv \varepsilon$. Furthermore, we shall assume [16], that $K$ is close to the value, defined by the Bragg's condition: $\left|K-\frac{2 \pi}{d}\right| \approx K$ $c^{2} K^{2} \approx \omega^{2} \varepsilon_{0}$. This case corresponds to a resonance of plane waves between the components $\vec{f}_{K, p}^{(E, H)}$ at $p=0,-1$ (these terms dominate in the system (7)). After eliminating the $\vec{f}^{(H)}$ variables, Eqs. (7) with respect to $\vec{f}^{(E)}$ take the form:

$$
\left[\begin{array}{cc}
K^{2}-\frac{\omega^{2}}{c^{2}} \varepsilon^{(0)} & -\frac{\omega^{2} \varepsilon^{(1)}}{c^{2}} \\
-\frac{\omega^{2} \varepsilon^{(-1)}}{c^{2}} & \left(K-\frac{2 \pi}{d}\right)^{2}-\frac{\omega^{2}}{c^{2}} \varepsilon^{(0)}
\end{array}\right]\left(\begin{array}{l}
f_{K, 0}^{(E)} \\
f_{K,-1}^{(E)}
\end{array}\right)=0
$$

where $\varepsilon_{l=0} \equiv \varepsilon^{(0)}, \varepsilon_{l= \pm 1} \equiv \varepsilon^{( \pm 1)}$. Putting the determinant of the system (8) equal to zero we obtain the dispersion relations $\omega_{ \pm}=\omega(K)$ . Two roots of this equation $\omega_{ \pm}$define the boundaries of the spectral band: at frequencies $\omega_{-}(K)<\omega<\omega_{+}(K)$ (band gap) the roots are complex and electromagnetic waves decay (Bragg's reflection); frequencies $\omega<\omega_{-}, \omega>\omega_{+}$correspond to propagating waves.

\section{Results and Discussion}

We shall confine ourselves to the case of propagation of electromagnetic radiation in a nonmagnetic superlattice with the two layers-elements (Si-layer and liquid crystal layer) per elementary cell. Concentration and dielectric permeability of the base material in the first and the second sublattice are denoted by $C_{1}^{(1)}, \varepsilon_{1}^{(1)}$ and $C_{2}^{(1)}, \varepsilon_{2}^{(1)}$ ( $\left.\varepsilon_{1}^{(1)}=11.7, \varepsilon_{2}^{(1)}=5.5\right)$ respectively. For admixture this quantities are denoted by $C_{1}^{(2)}, \varepsilon_{1}^{(2)}$ and $C_{2}^{(2)}, \varepsilon_{2}^{(2)}$. Simple transformations (with the account that $\left|\varepsilon^{(-1)}\right|=\left|\varepsilon^{(1)}\right|$ ) lead to the following relations for the refractive index $\mathrm{n} \equiv \mathrm{cK} / \omega$ of the studied system:

$$
n_{ \pm}^{2}\left(C_{1}^{(2)}, C_{2}^{(2)}\right)=\varepsilon^{(0)}\left(C_{1}^{(2)}, C_{2}^{(2)}\right) \pm\left|\varepsilon^{(1)}\left(C_{1}^{(2)}, C_{2}^{(2)}\right)\right| \cong \varepsilon^{(0)}\left[1 \pm \frac{\Delta \omega_{1}\left(C_{1}^{(2)}, C_{2}^{(2)}\right)}{\omega}\right]
$$

$\left(n_{+}^{2}-n_{-}^{2}\right) / 2 \varepsilon^{(0)} \cong \Delta \omega_{1} / \omega, \Delta \omega_{1}=\left|\omega_{+}-\omega_{-}\right|$- is the lowest band gap width. It follows from Eq. (9) that the quantity $\Delta \omega_{1}$ is determined by the corresponding coefficient of the Fourier expansion (5), which in this case is $\left|\varepsilon^{(1)}\right|$. The band gaps of higher orders are as well determined by corresponding Fourier-coefficients of the dielectric permeability.

$$
\begin{aligned}
& \varepsilon^{(0)}=\left(\varepsilon_{1}^{(1)} f_{1} a_{1}+\varepsilon_{2}^{(1)} f_{2} a_{2}\right) / d \\
& \left|\varepsilon^{(1)}\right|=\frac{1}{\pi}\left|\varepsilon_{2}^{(1)} f_{2}-\varepsilon_{1}^{(1)} f_{1}\right| \sin \pi a_{1} / d
\end{aligned}
$$

The functions $f_{1}\left(C_{1}^{(2)}, \frac{\varepsilon_{1}^{(2)}}{\varepsilon_{1}^{(1)}}\right)=1-C_{1}^{(2)}\left(1-\frac{\varepsilon_{1}^{(2)}}{\varepsilon_{1}^{(1)}}\right), f_{2}\left(C_{2}^{(2)}, \frac{\varepsilon_{2}^{(2)}}{\varepsilon_{2}^{(1)}}\right)=1-C_{2}^{(2)}\left(1-\frac{\varepsilon_{2}^{(2)}}{\varepsilon_{2}^{(1)}}\right)$ depend on the concentration of admixture layers and their relative dielectric permeability.

Figure 1 shows the concentration dependence of the refractive index $\mathbf{n}_{ \pm} \equiv \mathbf{c K} / \omega_{ \pm}$of the studied composite superlattice. It is readily seen, that the form of the corresponding surfaces has a non-monotone character if the dielectric permeability of both admixtures is $\varepsilon_{i}^{(2)} / \varepsilon_{i}^{(1)} \ll 1(i=1,2)$ (case a) or $\varepsilon_{i}^{(2)} / \varepsilon_{i}^{(1)} \gg 1(i=1,2)$. The dependence of $n_{+}$and $n_{-}$on $C_{1}^{(2)}$ and $C_{2}^{(2)}$ becomes monotonous in an other cases (b). The latter fact determines the behavior of the lowest band gap.

In Figure 2 the lowest energy gap width is plotted vs. the concentrations $C_{1}^{(2)}, C_{2}^{(2)}$ of admixture layers for a superlattice with alternating silicon and liquid-crystal layers. The energy gap $\Delta \omega_{1}$ 


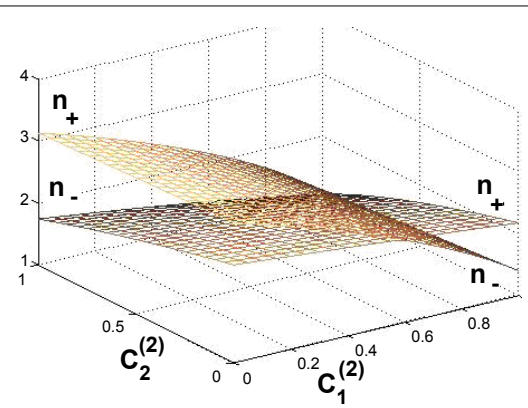

a)

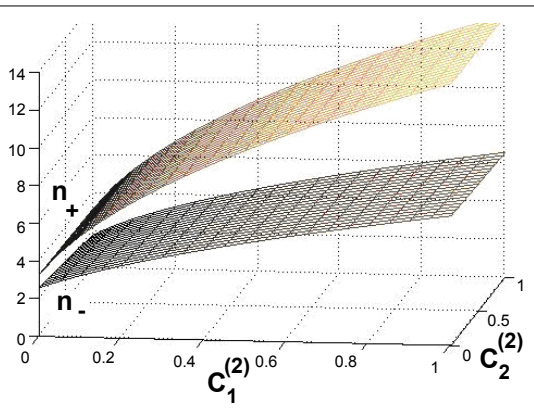

b)

Figure 1: Refractive index $\mathbf{n}_{ \pm} \equiv \mathbf{c K} / \omega_{ \pm}$of the composite superlattice (with alternating silicon and liquid-crystal layers) vs the concentrations of admixture layers: a) $\varepsilon_{1}^{(2)} / \varepsilon_{1}^{(1)}=0.1, \varepsilon_{2}^{(2)} / \varepsilon_{2}^{(1)}=0.2 ;$ b) $\varepsilon_{1}^{(2)} / \varepsilon_{1}^{(1)}=20, \varepsilon_{2}^{(2)} / \varepsilon_{2}^{(1)}=0.2 ; a_{1} / a_{2}=1$

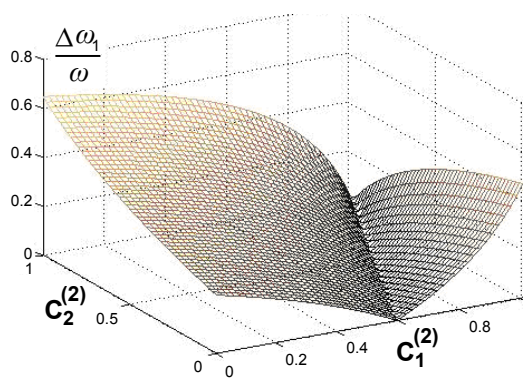

Figure 2: Relative width of the lowest band gap $\Delta \omega_{1} / \omega$ of the composite superlattice (with alternating silicon and liquid-crystal layers) vs the concentrations of admixture layers. Surface a) for the case $\varepsilon_{1}^{(2)} / \varepsilon_{1}^{(1)}=0.1, \varepsilon_{2}^{(2)} / \varepsilon_{2}^{(1)}=0.2$; surface b) for the case $\varepsilon_{1}^{(2)} / \varepsilon_{1}^{(1)}=20, \varepsilon_{2}^{(2)} / \varepsilon_{2}^{(1)}=0.2 ; a_{1} / a_{2}=1$. vanishes at $\varepsilon_{1}^{(1)} f_{1}=\varepsilon_{2}^{(1)} f_{2}$ for the case a) in Figure 2 .

\section{Conclusion}

Our present study shows that optical characteristics of an imperfect superlattice may be significantly altered as a result of transformation of its polariton spectrum due to presence of admixture layers. The developed theory is a basis for phenomenological description of a wide class of optical processes in nonideal multilayered systems. Formulas $(2-5,7)$ allow a numerical calculation of the concentration dependence of relevant optical characteristics. The essential quantities governing the propagation of electromagnetic waves through the studied media are the refractive indices, the photon gap width and the directly measured quantities, which they define (for example, the light transmission coefficient). Graphic representation of $n_{ \pm}, \Delta \omega / \omega\left(C_{1}^{(2)}, C_{2}^{(2)}\right)$ (Figure 1 and 2) shows, that for the considered binary systems the character of the concentration dependence is different in different concentration intervals. The study carried out by authors [24] shows that optical characteristics of the nonideal photonic crystals may vary due to transformation of its photon modes spectrum caused by the presence of admixture elements. The case of nonideal multilayered systems with bigger number sublattices and components of alien layers allows even for a greater variety in behavior of the refractive index and the gap width. This circumstance considerably widens the opportunities for modeling such composite materials with prescribed properties.

\section{References}

1. Zhang C, Hirt DE (2007) Layer-by-layer self-assembly of polyelectrolyte multilayers on cross-section surfaces of multilayer polymer films: A step toward nano-patterning flexible substrates. Polymer 48: 6748-6754.

2. Liu H, Zhu SN, Dong ZG, Zhu YY, Chen YF, et al. (2005) Coupling of electromagnetic waves and superlattice vibrations in a piezomagnetic superlattice: Creation of a polariton through the piezomagnetic effect. Phys Rev B 71: 125106

3. Baraban LA, Lozovski VZ (2004) Reflection and absorption of light by a thin semiconductor film. Opt Spectrosc 97: 810-816.

4. Rumyantsev VV, Shunyakov VT (1992) Exciton-polariton dispersion in ultrathin atomic cryocrystals. Physica B 176: 156-158.

5. Figotin A, Vitebsky I (2001) Nonreciprocal magnetic photonic crystals. Phys Rev E 63: 066609.

6. Lyubchanskii IL, Dadoenkova NN, Lyubchanskii MI, Shapovalov EA, Lakhtakia A, et al. (2004) One-dimensional bigyrotropic magnetic photonic crystals. Appl Phys Lett 85: 5932-5934

7. Belotelov VI, Kotov VA, Zvezdin AK (2005) Abstracts of the International Conference on Functional Materials. Partenit, Cremea, NASU: Kiev, Ukraine.

8. Ha YK, Yang YC, Kim JE, Park HY, Kee C-S, et al. (2001) Tunable omnidirectiona reflection bands and defect modes of a one-dimensional photonic band gap structure with liquid crystals. Appl Phys Lett 79: 15-17.

9. Yi Y, Bermel P, Wada K, Duan X, Joannopoulos JD, et al. (2002) Tunable multichannel optical filter based on silicon photonic band gap materials actuation. Appl Phys Lett 81: 4112-4114.

10. Tolmachev VA, Perova TS, Berwick K (2003) Design Criteria and Optica Characteristics of One-Dimensional Photonic Crystals Based on Periodically Grooved Silicon. Appl Optics 42: 5679-5683.

11. Tolmachev VA (2005) Tuning of the photonic band gaps and the reflection spectra of a one-dimensional photonic crystal based on silicon and a liquid crystal. Opt Spectrosc 99: 765-769. 
Citation: Rumyantsev VV, Schwartzman A (2013) Peculiarities of Propagation of Electromagnetic Excitations through Nonideal 1d Photonic Crystal. J Electr Electron Syst 2: 109. doi:10.4172/2332-0796.1000109

Page 4 of 4

12. Tolmachev VA, Perova TS, Astrova EV (2008) Thermo-tunable defect mode in one dimensional photonic structure based on grooved silicon and liquid crystal. Phys Status Solidi (RRL) 2: 114-116.

13. Bass FG, Bulgakov AA, Tetervov AP (1989) High-Frequency Properties of Semiconductors with Superlattices; Moscow, Russia.

14. Pokatilov, EP, Fomin, VM, Beril SI (1990) Vibrational Excitations, Polarons, and Excitons in Multilayer Systems and Superlattice. Shtiintsa, Chisinau, Moldova.

15. Tyu NS (1994) Local field effects and tensors of dielectric permeability in organic superlattices. Solid State Commun 90: 667-675.

16. Yariv A, Yeh P (1984) Optical Waves in Crystals: Propagation and Control of Laser Radiation. Wiley, New York, USA.

17. Awasthi SK, Ojha SP (2008) Wide-Angle Broadband Plate Polarizer with 1D Photonic Crystal. PIER 88: 321-335.

18. Banerjee A (2009) Enhanced Refractometric Optical Sensing By Using OneDimensional Ternary Photonic Crystals. PIER 89: 11-22.
19. Parmenter RH (1955) Energy Levels of a Disordered Alloy. Phys Rev 97: $587-$ 698

20. Dargan TG, Capaz RB (1997) Koiler Belita Braz J Phys 27/A: 299-304.

21. Ziman JM (1979) Models of disorder. The theoretical physics of homogeneously disordered systems, John Willey \& Sons, Inc, New York, USA.

22. Mezrag F, Aouina NY, Bouarissa NJ (2006) Optoelectronic and dielectric properties of $\mathrm{GaAs}_{x} \mathrm{Sb}_{1-x}$ ternary alloys. J Mater Sci 41: 5323-5328.

23. Los' VF (1987) Projection operator method in the theory of disordered systems. I. Spectra of quasiparticles Theor Math Phys 73: 85-102.

24. Rumyantsev VV, Fedorov SA, Gumennyk KV (2011) Peculiarities of Band Gap Width Dependence Upon Concentration of Admixtures Randomly Included in 1D Photonic Crystal. Photonic Crystals: Optical Properties, Fabrication and Applications. Nova Science Publishers, Inc, New York, USA. 